\title{
The angular distribution of ion flux around an ionospheric satellite
}

\author{
URI SAMIR \\ Space Physics Research Laboratory, University of Michigan, \\ Ann Arbor, Michigan, U.S.A. \\ and \\ Department of Environmentel Sciences, \\ Tel-Aviv University, Ramat-Aviv, Israel \\ and \\ E. J. Mater and B. E. Troy, Jr. \\ Goddard Space Flight Center, Laboratory for Planetary Atmospheres, \\ Greenbelt, Maryland 20771, U.S.A.
}

(Received 3 May 1972)

\begin{abstract}
Ion measurements from the Explorer 31 satellite were used to determine the angular distribution of ions around the satellite. The ion distributions were compared with the electron distributions measured on the same satellite. It is shown that the electron currents (fluxes) in the wake are always larger than the ion currents for a wide domain of plasma parameters. A quantitative comparison of the ion fluxes with a neutral approximation model for the angular distribution is considered. It is found that the ion fluxes become progressively larger than the corresponding computed neutral fluxes as the angle attack increases from $0^{\circ}$ towards $180^{\circ}$.
\end{abstract}

\section{INTRODUCTION}

THE supersonic/hypersonic motion of a satellite creates a region of disturbance around the satellite. The disturbed region reaches large distances downstream but is most pronounced close to the satellite surface. Essentially, the very close region behind the satellite downstream (the 'wake-region') is depleted unequally of both electrons and ions. The amount of particle depletion in the wake region depends on the fundamental plasma parameters

$$
R=R_{o} / \lambda_{D}, \quad T=\frac{T_{e}}{T_{+}}, \quad S=\frac{V_{s}}{\sqrt{\frac{2 k T_{s}}{M_{+}}}}, \quad \phi=\frac{e \phi_{s}}{k T_{s}},
$$

where $R_{o}=$ radius of satellite, $\lambda_{D}=$ Debye length of the undisturbed ambient plasma, $T_{a}$ and $T_{+}=$electron and ion temperature respectively, $V_{\mathrm{s}}=$ satellite velocity, $M_{+}=$ionic mass, $\phi_{s}=$ satellite potential, $k=$ Boltzmann's constant, and $e=$ electronic charge.

Numerous theoretical studies are available in the literature dealing with various aspects of the phenomena involved in the interaction between a satellite and the ionospheric spaceplasma. Of the most recent review papers we cite GurEvich et al. (1970), LrU (1969) and VAGLTo et al. (1972).

On the other hand the amount of in situ observations regarding both angular and axial charged particle distribution in the satellite surroundings is meagre. Among these, most of the available results refer to the angular distribution of electrons in the nearest vicinity to the satellite surface (e.g. SAMIR and WrenN, 1969). Very 
little, however, is available for either the angular (e.g. Hoffman, 1969) or the axial (e.g. Troy et al., 1970) distribution of ions around an ionospheric satellite.

It is the purpose of this paper to present and briefly discuss some results regarding the ion fluxes around the ionospheric satellite Explorer 31. Ambient ion and electron measurements are used to derive ionospheric parameters, which are then related to the observed ion fluxes.

\section{EXPERIMENTAL}

The Explorer 31 satellite was launched on 29 November 1965 into a near-polar orbit (inclination $80^{\circ}$ ), with a perigee of $500 \mathrm{~km}$ and an apogee of $3000 \mathrm{~km}$. It was spin-stabilized, with the spin axis oriented approximately perpendicular to the orbital plane during the periods covered by this paper. The satellite is approximately cylindrical in shape having a radius of about $40 \mathrm{~cm}$.

The ion measurements were made by a planar Retarding Potential Analyser (RPA). The RPA is mounted in the equatorial plane of the satellite, with its aperture normal situated perpendicular to the satellite spin axis.

In this location, the RPA moves through a full $360^{\circ}$ in one spin period $(\sim 20 \mathrm{sec})$, looking first forward along the velocity vector and then backward into the wake. The ion flux to the satellite can then be measured as a function of the angle $\theta$ between the trap normal and the velocity vector $\left(\theta=0\right.$ forward, $180^{\circ}$ in the wake). The ambient ion density and temperature are determined when the RPA is looking forward. A similar RPA, programmed for electron measurements, determines the satellite potential and the ambient electron temperature. The data are corrected for photoelectron current when the Sun is shining into the aperture.

Details of design, operation and data analysis for the Explorer 31 RPA's are discussed elsewhere (DoNLeY, 1969).

\section{Results and Discussion}

Figure 1 shows an example of the general trend of the ion flux (ion current) with time as the spacecraft rotates (or spins) around its axis. In this example the amplitude of the ion flux modulation changes from a factor of 30 to a factor of 600 as the satellite encounters different plasma parameters. It is seen that the upper limit of the modulation, proportional to the product of the ion density and the spacecraft

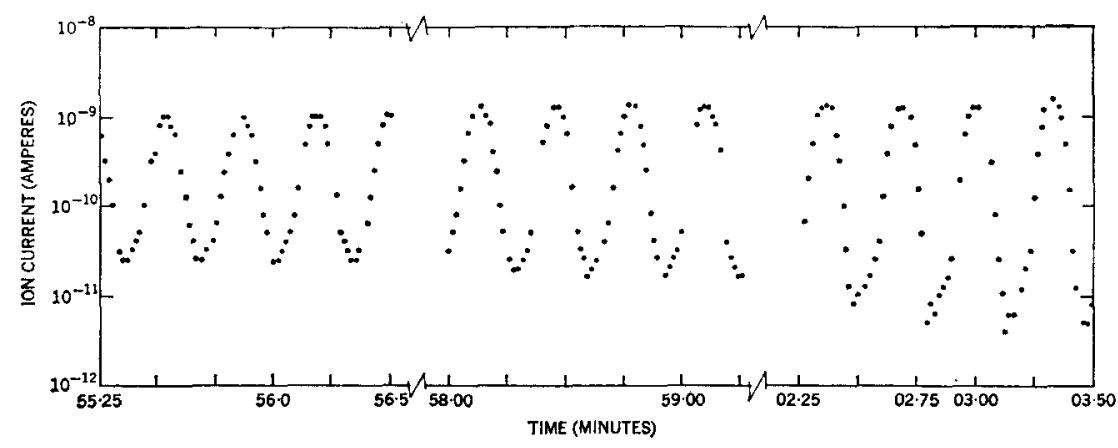

Fig. 1. Example showing the time dependence of ion-flux for pass 482 . 
velocity, is similar throughout the pass (within 20 per cent), and the larger amplitudes reflect variations of the values of the minima. The amount of ion depletion in the wake region varies with the plasma parameters which depend mainly on the plasma temperature and composition.

By using the ion flux data such as shown in Fig. 1, along with the corresponding values of the angle of attack $\theta$, it is possible to determine the flux $I_{+}$as a function of $\theta$ through the complete satellite spin. This has been done for six passes selected for a wide range of altitudes and plasma parameter values; the resulting flux values are given in Table 1. Column 5 lists the flux values $I_{+}(F)$ taken in the forward direction, i.e. when $\theta=0^{\circ}$. Values $I_{+}(\theta)$ at $\theta=90^{\circ}, 100^{\circ}, 115^{\circ}, 130^{\circ}, 145^{\circ}$ and $165^{\circ}$ are shown in columns 6-11, and are normalized to $I_{+}(F)$. It is seen that the amount of ion flux depletion in the wake with respect to ambient $\left[I_{+}(F)\right]$ increases at lower altitudes and decreases at higher altitudes. A similar behavior was shown by SAMIR and WRENN (1969) to exist for the electrons using observations from the same satellite.

Table 2 lists the corresponding ambient data for the same sets of passes covered in Table 1. The first columns show the measured values of $T_{e}, T_{+}, \phi_{s}$ and

$$
N_{+}=\left[\mathrm{H}^{+}\right]+\left[\mathrm{He}^{+}\right]+\left[\mathrm{O}^{+}\right]
$$

as well as the given satellite velocity $V_{s}$ and the average ionic mass $M_{+}$computed from the three individual ion densities. The last four columns give the values of the fundamental plasma parameters derived from the ambient measurements.

Selecting cases of comparable $R, \phi$ and $T$ values but different values of $S$ shows that the amount of ion current depletion in the wake increases with increasing values of $S$ and $\theta$. For example, in pass 459 (altitude range: $834-791 \mathrm{~km}$ ) the value of $I_{+}(130) / I_{+}(F) \simeq 3.2 \times 10^{-2}$ while in pass 683 (altitude range: $527-521 \mathrm{~km}$ ) that ratio is $2 \cdot 2 \times 10^{-4}$, i.e. smaller by about two orders of magnitude. The principal difference between these two cases being $S=3.8$ for the former and $S=6 \cdot 0$ for the latter.

Moreover, $\quad \frac{I_{+}(100)}{I_{+}(130)} \simeq 2 \cdot 2$ for $S=3 \cdot 8$ and $\frac{I_{+}(100)}{I_{+}(130)} \simeq 17$ for $S=6 \cdot 0$,

hereby indicating the significant difference in the slopes of the $I_{+}(\theta)$ curves.

Figure 2 shows the variation of the normalized measured ion and electron currents with angle of attack $(\theta)$ for $90^{\circ} \leq \theta \leq 180^{\circ}$ for three passes. The electron data used are from SAMrR and JEW (1972), and are averaged over the whole pass. The ion data are taken from Table 1, and are averaged over the altitude range for which ion data are available as shown in the table. The fundamental plasma parameters (last four columns in Table 2) may be compared for the two sets of data. Corresponding to the ions, we have by averaging the data in Table 2 , for pass $393, R=20 \cdot 3, T=1 \cdot 1$, $\phi=-4 \cdot 6$ and $S=4.8$; for pass $482, R=18 \cdot 9, T=1 \cdot 06, \phi=-3 \cdot 2$ and $S=-3 \cdot 9$; and for pass $683, R=20 \cdot 8, T=1 \cdot 23, \phi=-3 \cdot 6$ and $S=5 \cdot 8$. For the electrons, we have from SAMUR and JEW (1972) for pass $393, R=7 \cdot 8, \phi=-5 \cdot 5, S=3 \cdot 6$; for pass $482, R=9 \cdot 5, \phi=-3 \cdot 8, S=3 \cdot 7$; and for pass $683, R=43, \phi=-5 \cdot 1$, $S=5 \cdot 6$. The differences between the electron and ion parameters in pass 393 are due to the fact that the ion parameters represent an average over the altitude range $695-786 \mathrm{~km}$ whereas the electron parameters are averaged over the altitude range $696-1071 \mathrm{~km}$. 
URI SAMIR, E. J. MAIER and B. E. Troy, JR.

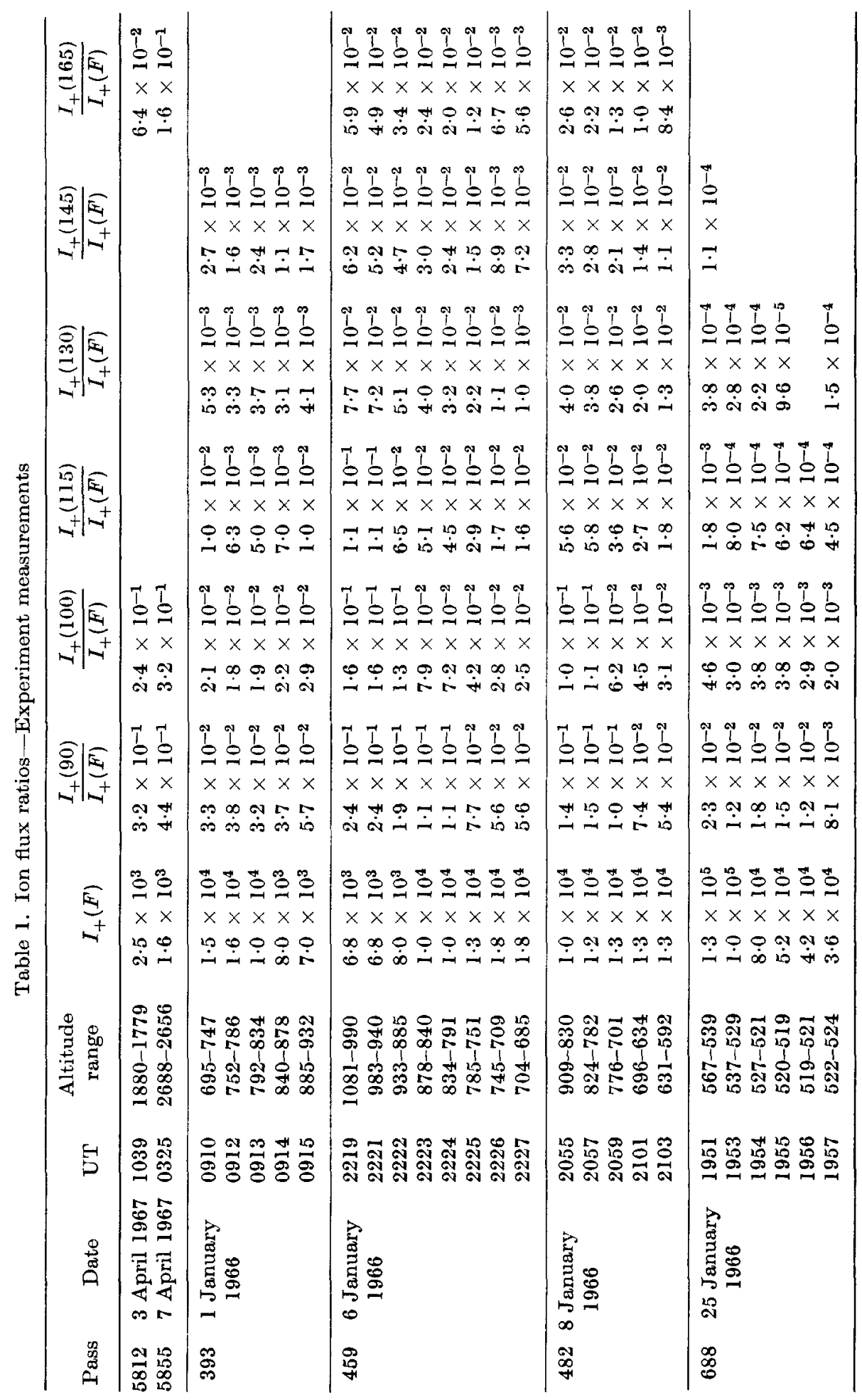


Table 2. Ionospheric plasma properties and revelant plasma parameters for the above passes.

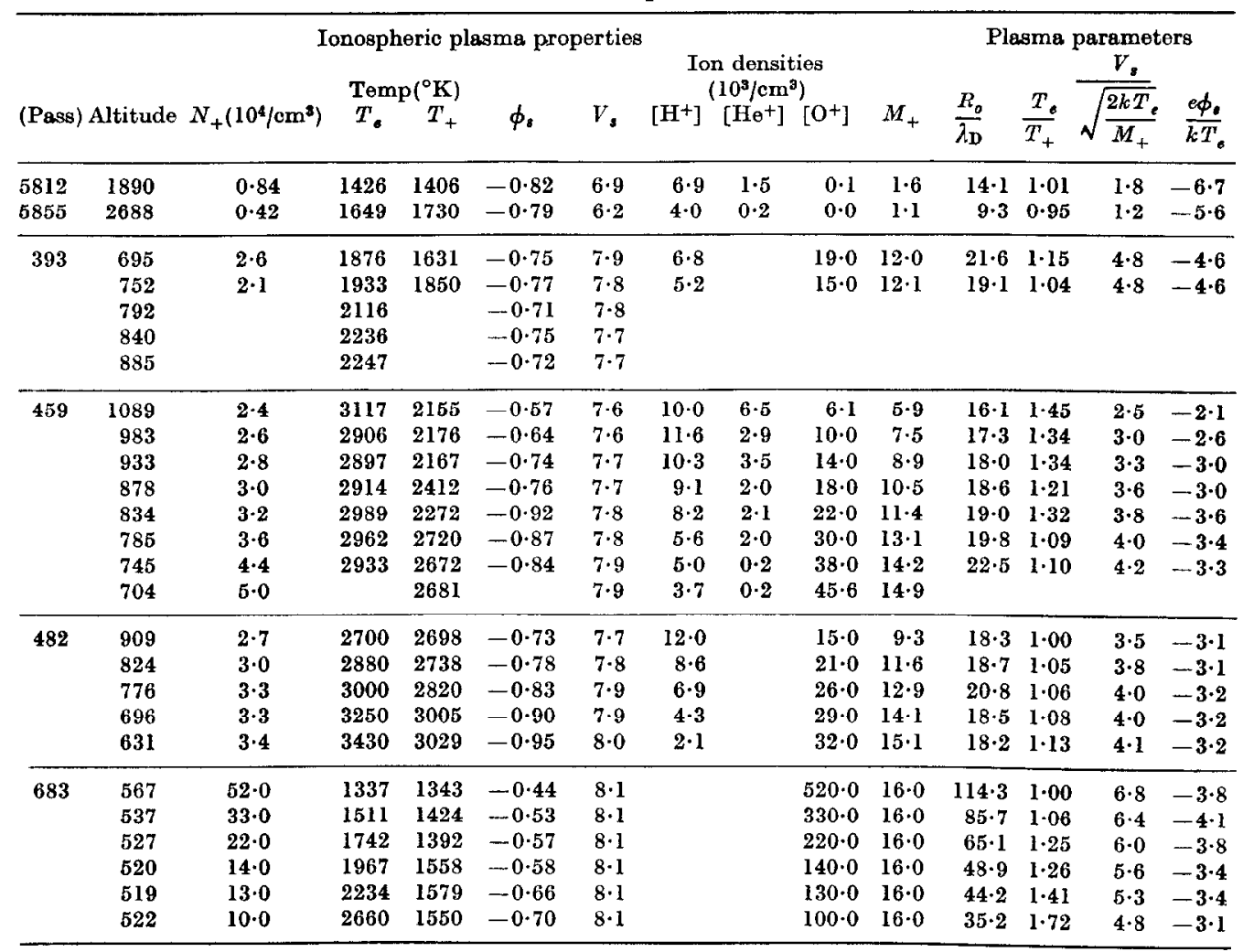

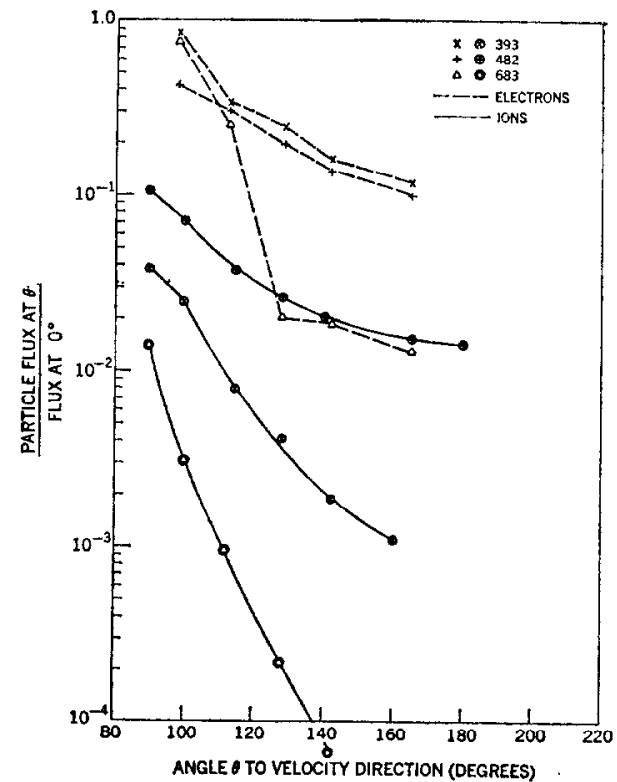

Fig. 2. Variation of normalized measured ion and electron currents with angle of attack (0) for $90^{\circ} \leq \theta \leq 180^{\circ}$. 
It is seen that the amount of ion depletion in the wake is always greater than the corresponding electron depletion. Although this could have been qualitatively anticipated it is the first time (to the best of the authors' knowledge) that the differences between $I_{+}(\theta)$ and $I_{\theta}(\theta)$ in the wake region are demonstrated quantitatively using in situ observations. This result is generally in accord with theory (e.g. LIU, 1969; GUREvich et al., 1970).

Figure $3(a, b)$ show a comparison between the measured normalized ion current and the corresponding theoretical computation treating ions in the classical neutral approximation generally referred to as $F(S)$. Figure $3($ a) refers to passes 393 and 482 and Fig. $3(\mathrm{~b})$ to passes $459,683,5812,5855$. Note that data from pass 482 was divided into two altitude intervals, $482 \mathrm{~A}$ from $909-830 \mathrm{~km}$ and $482 \mathrm{~B}$ from $631-592 \mathrm{~km}$. For the purpose of the neutral approximation computation we define

$$
F\left(\theta, M_{i}\right)=\frac{F(S \cos \theta)}{F_{o}(S)} .
$$

Our computed neutral flux ratio is then:

$$
J(\theta)=\frac{N\left(\mathrm{H}^{+}\right) F(\theta, 1)+N\left(\mathrm{He}^{+}\right) F(\theta, 4)+N\left(\mathrm{O}^{+}\right) F(\theta, 16)}{N\left(\mathrm{H}^{+}\right)+N\left(\mathrm{Ie}^{+}\right)+N\left(\mathrm{O}^{+}\right)} .
$$

This was done to estimate the quantitative difference between the observed ion flux and the neutral flux predicted by the frequently used $F(S \cos \theta)$. That is, $J(\theta)$ would represent the flow of neutral particles having the same mass distribution as we measured for the ion species. This assumes that the separate ion species are noninteracting. The comparison shows quantitatively the influence of the potential field and collective phenomena on the ion motion not considered by the neutral approximation. This is of critical significance very close to the satellite. The $J(\theta)$ plots show values lower than those of the corresponding measured ion fluxes. This might be expected on the basis of a net negative charge in the wake of the spacecraft. The
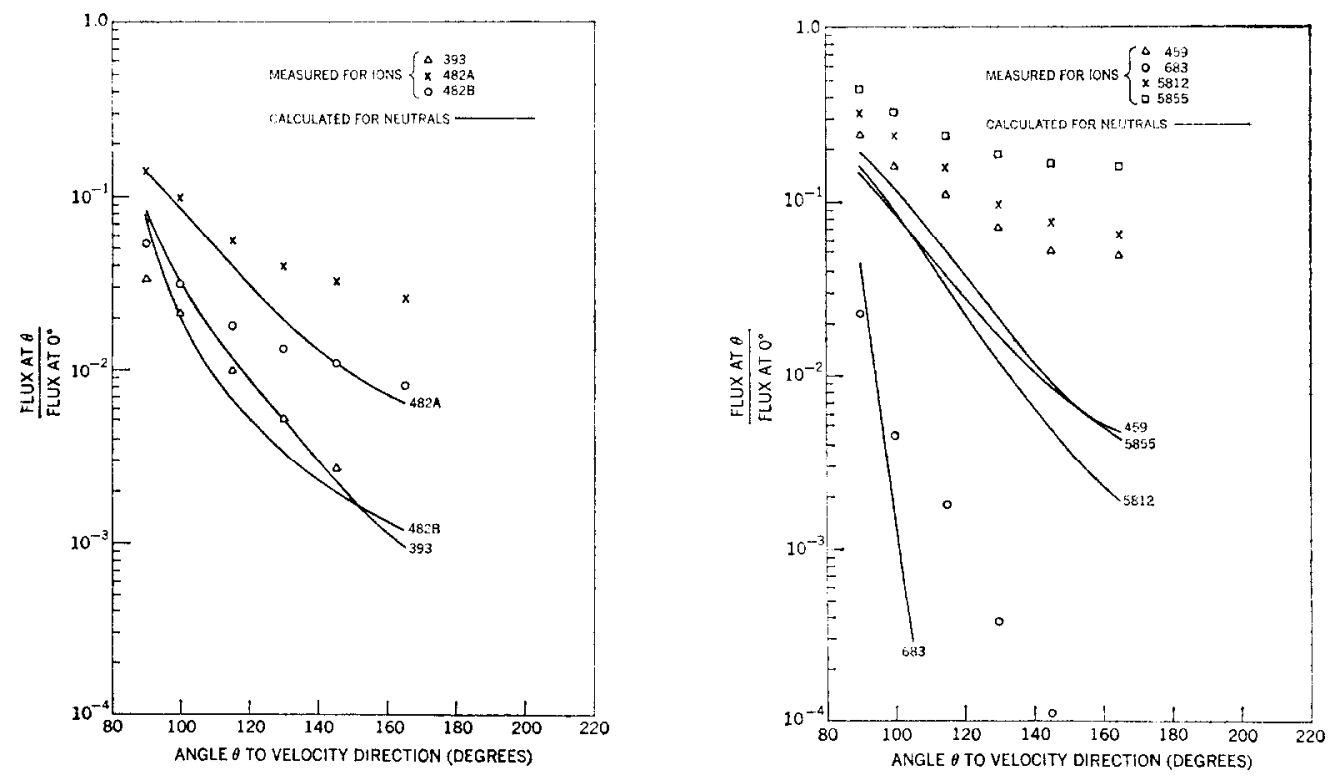

Fig. 3. Comparison between the normalized measured ion current, and the corresponding computed neutral particle flux ratios. 
resulting inward acceleration of ions leads to ion velocities and thus fluxes larger than those present for neutral particles at the same location. SERBU and MaIER (1970) did a similar comparison for data obtained at higher altitudes. They found that there was good agreement with the neutral approximation using the $F(S \cos \theta)$ function in the angle range from $0^{\circ}$ to $90^{\circ}$ in a 100 per cent $\mathrm{H}^{+}$plasma.

We have also computed the neutral flux ratios assuming a single species of mass $M_{+}$in the calculation of $S$. This yielded wake depths far greater than those presented in Fig. 3. Because of the strong mathematical dependence of $F(S)$ on the ion mass, it seems more physically sound to use the linear superposition method of equation (1) rather than to evaluate the wake depth using an average mass. The physical implication of this is that the hydrogen ions play the major role in determining the ion depletion in the wake.

Similar reasoning was adopted by GUREvich et al. (1970) in their comparison between the SAMIR and WRENN (1969) results and their theoretical wake model.

In a recent paper studying (in the laboratory) the flow of rarefied plasma around a body, BogashCHENKo et al. (1971) compared their experimental results for the near-wake with the GuREvicH et al. (1970) wake model.

It was shown that the GUREvICH et al. (1970) neutral approximation applies for the ions in the wake at distances $Z \sim 2.8 R_{o}$ on the axis. Near the body's surface, however, the ion current was about one to two orders of magnitude larger than the corresponding values computed for the 'neutral approximation'.

Although the above comparison had flow parameters not identical to ours, their results are generally in line with those presented in Fig. 3 for the difference between the measured ion fluxes and computed 'neutral approximates'.

Employing the BogashCHENKo et al. (1971) study lends general support to our argumentation about the hydrogen ions being of decisive role in determining the ion-wake.

In summary, we have used in situ observations to perform a quantitative comparison between ion and electron depletions in the wake of the Explorer 31 satellite for a wide range of plasma parameters. In all cases the ion depletion was greater than the electron depletion. We have also quantitatively demonstrated the discrepancies encountered in using the 'neutral approximation' for ions in the very near wake.

\section{REFERENCES}

Bogashcheinko I. A., GURevich A. V., Salimov R. A. and Eidelman YU. I. DoNLey J.

Gurevich A. V., Pitaevsei L. P. and SMIRnova V. V.

HoffmaN J. $\mathrm{H}$.

Lru V. C.

SAMir U. and WrenN G. L.

SAMIR U. and JEW $H$.

Serrbu G. P. and Mater E. J. R.

Troy B. E., JR., Medved D. and SAMTR U.

Vaglio-Laurin R. and Miller G.
1971 Soviet Phys. J.E.T.P. 32, 841.

1969 Proc. $I E E E$ 5'\%, 1061

1970 Soviet Phys.-Uskpekhi p. 595

1967 Proc. IEEE 57, 1063.

1969 Space Sci. Rev. 9, 423.

1969 Planet. Space Sci. 17, 693.

$1972 J$.geophys. Res. (to be published.)

$1970 \quad J$. geophys. Res. 75, 6102

$1970 J$. astron. Sci. 18, 173.

1972 Rarefied Gas Dynamics. 